\title{
Chapter 6 \\ Considering Social Play in Primates: A Case Study in Juvenile Tibetan Macaques (Macaca thibetana)
}

\author{
Jessica A. Mayhew, Jake A. Funkhouser, and Kaitlin R. Wright
}

\subsection{Introduction}

The evolutionary origins and adaptive value of animal play behavior have long been contemplated. Social play in gregarious animals is a multidimensional topic that has long been debated, insufficiently investigated, and a source of enigmatic questions regarding its development, relationship to cognition, and adaptive value. Play has been characterized as the most sophisticated manifestation of communication (Bekoff 1972; Bekoff and Allen 1997; Burghardt 2005; Fagen 1981; Pellis and Pellis 2009), which is partially why it is challenging to study. Beyond its incorporation into species' activity budgets, researchers have encountered multiple logistical and theoretical stumbling blocks, including difficulty in operationalizing definitions, identifying multiple juveniles and their varied social relationships, keeping pace with changes in interaction tempo and player composition, quantifying the behaviors observed and their short- and long-term costs and benefits, and determining an individual's motivation and intention to engage with others in this specific manner. Nevertheless, social play warrants attention because of its inherent complexity,

\footnotetext{
J. A. Mayhew ( $\square)$

Department of Anthropology and Museum Studies, Central Washington University, Ellensburg, WA, USA

Primate Behavior and Ecology Program, Central Washington University, Ellensburg, WA, USA e-mail: MayhewJ@cwu.edu

J. A. Funkhouser

Primate Behavior and Ecology Program, Central Washington University, Ellensburg, WA, USA

Department of Anthropology, Washington University in St. Louis, St. Louis, MO, USA

e-mail: jakefunkhouser@wustl.edu

K. R. Wright

Primate Behavior and Ecology Program, Central Washington University, Ellensburg, WA, USA

e-mail: wrightkr@uw.edu
} 
perceived contributions to an individual's fitness, communicative content, and interspecies variability.

In this chapter, we provide a brief overview of play behavior, with special attention to macaques, and propose some considerations to others interested in studying play. To emphasize some of these points, we provide an example where we consider the multiple factors characterizing the social play behavior of the 2017 Yulingkeng A1 infant and juvenile Tibetan macaques (Macaca thibetana) at Mt. Huangshan, China. Additionally, we map their positions in a social play network and use this foundation to generate hypotheses regarding their future network positions. We encourage future studies to address how the construction of these juvenile relationships contributes to an individual's overall group position and the formation of adult relationships and the potential adaptive advantage play provides.

\subsection{Play Behavior: An Overview}

The diversity in the form and content of play has generated discussion about the proximate and ultimate costs/benefits and whether generalizations about this behavior can be made across taxa. There are multiple definitions of play emphasizing either structure or function (e.g., Bekoff and Allen 1997; Fagen 1981; Martin and Caro 1985), but the development of five descriptive criteria by Burghardt (2005) has provided researchers the parameters to identify play as distinct from other common behaviors in a repertoire. For a behavior to be labeled "playful," Burghardt (2005) proposes it should (1) have a limited immediate function, (2) be endogenous, (3) have structural or temporal properties that are different from "serious" behaviors,

(4) be flexibly exercised and not stereotypical, and (5) be performed in a relaxed field (i.e., free of stress or social/physical pressures). Guided by these criteria, researchers now have a foundation to tackle more complex questions of how and why animals play.

Play is often broadly categorized as solitary, object, and social (Bekoff and Byers 1981; Fagen 1981), but these categories are not mutually exclusive. Solitary play typically includes intense or sustained locomotor movements performed alone, e.g., the running and gamboling of young ungulates. Object play can be solitary or social and includes the manipulation of an object for no immediate benefit. Object play is commonly observed in carnivores, in which predatory movements, such as grabbing and shaking, are performed on a non-prey item (Burghardt 2005). Primates also engage in object play from stick carrying in chimpanzees (Pan troglodytes) (Kahlenberg and Wrangham 2010), stone handling in Japanese macaques (M. fuscata, Nahallage et al. 2016; Shimada 2006, 2010), to the manipulation of one's environment in functional object substitution, sparking discussion about pretense, imagination, and theory of mind (Gómez and Martín-Andrade 2002). Social play is identified as being interactive and occurs between two or more conspecifics who may influence one another's actions (Thompson 1996). Social play often includes aggressive behaviors, such as biting and wrestling (Burghardt 2005), but tends to be reciprocal between partners (Fagen 1981). 
The type and exhibition of play depends on the species observed. Typically, the occurrence of play increases throughout juvenilehood but then tapers off at sexual maturity where activity budgets and individual priorities shift toward competition and reproductive resources. In birds, orders with more altricial species tend to engage in more play than orders with mostly precocial species (Ortega and Bekoff 1986). Raptors (e.g., eagles and hawks) engage in object play (Ortega and Bekoff 1986), young herring gulls (Larus argentatus) have been observed performing "drop-catch" behavior with clams and nonfood objects over hard substrates (Gamble and Cristol 2002), and ravens (Corvus corax) slide down snowy inclines and hang upside down from tree branches (Heinrich and Smolker 1998). Members of the family Canidae, including wolves, coyotes, foxes, and jackals, engage in solitary, object, and social play. Of this group, domestic dogs (Canis familiaris) are the most familiar play partners to humans and even use play signals that can be readily understood and responded to (e.g., the play bow) (Bekoff 1974). Cetacean play also takes on multiple forms, including the creation of play objects in the form of bubbles (Hill et al. 2017; Jones and Kuczaj 2014). For elephants (Loxodonta africana), play occurs throughout the life course on land and in water, and object play can be observed by individuals of all ages (Lee and Moss 2014). Guided by Burghardt's criteria, play can also be identified in animals that are not commonly regarded as being playful, including cichlid fish (Tropheus duboisi, Burghardt et al. 2014), Nile softshell turtles (Trionyx triunguis, Burghardt et al. 1996), octopus (Octopus dofleini, Mather and Anderson 1999), and even spiders (Pruitt et al. 2012).

Social play intrinsically involves partner cooperation, complex communication, and learning, and these are critical variables to investigate if we are to understand the cognitive and social development in young individuals (Bekoff and Allen 1997; Palagi et al. 2007). The functional significance of this suite of "nonserious" social behaviors remains elusive yet intriguing to ethologists; nevertheless, play has been noted to nourish the physical, social, and cognitive aspects of an individual (see Palagi 2018 for review). Namely, play functions to increase and maintain physical fitness or motor performance (Byers and Walker 1995; Fontaine 1994; Martin and Caro 1985), refines social skills and increases behavioral flexibility (Baldwin and Baldwin 1974; Brown 1988; Fagen 1984), and may be an important context that enhances cognitive skills in which an individual learns to identify and respond to the intentions of others through social cues (Bekoff 1972; Bekoff and Allen 1997; Palagi et al. 2007). These types of interactions serve as an opportunity to accrue and refine adult social skills and enhance behavioral flexibility (Baldwin and Baldwin 1974; Brown 1988; Fagen 1984). The unpredictable nature of this intimate social exchange challenges the participants to literally "think on their feet" as the interaction occurs, which imposes some inherent risk, but sharpens social tactics and reactions. In general, play is expected to occur more frequently with a partner that is evenly matched in skills and ability (M. fuscata, Kulik et al. 2015) as well as with individuals that are likely to be frequently encountered in adulthood (e.g., Maestripieri and Ross 2004). Pellis and Pellis (1996) hypothesize that rough-andtumble play influences the developmental of dominance relationships in postpubertal juveniles (i.e., subadult), especially in male-male play bouts, through testing a play 
partner's strength. Intense or more aggressive behaviors, such as bite or chase, could be used to create or maintain a competitive edge in a play bout (e.g., Gorilla gorilla, van Leeuwen et al. 2011) that serves as practice for future, more aggressive interactions requiring the defense or acquisition of resources. Therefore, playing at increased rates and with a diversity of partners is likely an adaptive strategy during juvenile development to enhance one's success in "serious" behaviors later in life. However, with variable social systems (between or within species), it can be expected that juvenile play rates, relationships, and partner diversity depend on the life stage (i.e., the priorities of an individual at different ages) and group composition (i.e., social partner diversity and range restrict group demography).

In addition to potentially preparing a juvenile for future social experiences (Burghardt 2005; Fagen 1981), researchers can study play in attempt to predict the context, quantity, and dyadic relationship quality of future social relationships between group members (e.g., Pellis et al. 2019). Play partner choice tends to reflect the adult social partners of cooperative, competitive, and reproductive relationships experienced in adulthood (e.g., Maestripieri and Ross 2004). At its core, play builds upon reciprocity and turn-taking between players; without reciprocity and clear communication it is difficult to maintain the interaction and playful context. Partner selection is thus important, as regularly playing against larger, stronger individuals may mean being disadvantaged more frequently. Similarly, selecting a smaller, less skilled, or younger opponent might mean you possess a more frequent advantage but may require more self-handicapping (e.g., Cebus apella, Lutz and Judge 2017; Macaca mulatta, Yanagi and Berman 2014b). Interactions with larger age disparities may require increased communication, i.e., play signaling, to maintain a playful context. For example, self-handicapping behaviors (e.g., adopting a supine position) are positively associated with play signaling in domestic dogs (Canis lupus familiaris, Ward et al. 2008), and gorillas often pair an open-mouth face with more intense play behaviors, such as chasing, potentially to maintain an equitable level of cooperation between play partners (van Leeuwen et al. 2011).

In the order Primates, there is marked interspecific variation in play, and even within a species, the frequency in which an individual engages in play is not uniform. Teasing apart the contributing individual- and group-level variables for these differences is no small task, and these intrinsic and external factors can be multidimensional. For example, young infants may be physically handicapped when engaged in play with larger juveniles due to their small size, limited motor coordination, and unrefined social skills. However, young infants may also be physically restricted or actively discouraged by their mothers from participating in play (e.g., the mother has a restrictive rearing style or is low-ranking in the group). Other variables, including group social organization, structure, style, and status (Ciani et al. 2012; Fagen 1981; Maestripieri 2004; Thierry 2007), an individual's opportunity (Panksepp and Beatty 1980), prior experience (Cloutier et al. 2013), personality (Lampe et al. 2017; Pellis and McKenna 1992), and brain chemistry (Siviy et al. 2011) may also influence an individual's participation in and style of play. Although it has been acknowledged that play typically declines in frequency with age, some primates continue to engage in play beyond sexual maturity, maintaining playful 
relationships with juveniles and even playing with other adults (Pellis and Iwaniuk 2000; e.g., Lemur catta, Palagi 2009; Macaca tonkeana, Ciani et al. 2012; Pan paniscus, Palagi 2006; Pan troglodytes, Yamanashi et al. 2018; Theropithecus gelada, Mancini and Palagi 2009). The motivation to continue playing into adulthood likely differs depending on a variety of factors, including the degree of social tolerance in the species (e.g., despotic vs. egalitarian society), species-specific affiliative patterns of behavior, and the evolved communicative mechanisms to maintain a playful context.

\subsection{Macaque Play}

Macaca spp. vary widely in their geographical distribution, but the genus shares certain foundational similarities in social organization; for example, they can be found living in female philopatric, multi-male, multi-female groups with overlapping home ranges. However, the differing geographic distribution and phylogeny of the genus has resulted in interspecific variation in patterns of affiliation, reconciliation, dominance, aggression, nepotism, and temperament (Thierry 1985, 1990; Thierry et al. 2000). From this variation, the social organization of macaques is typically regarded as a continuous, four-grade scale of dominance style: species occupying the first grade are considered hierarchical and nepotistic and those occupying the fourth grade are considered more tolerant or egalitarian (Thierry et al. 2000). Dominance style can be defined as the dominance relationships, categorized by agonistic interactions, within dyads in a social group (Thierry et al. 2000). A difference in dominance style between primate taxa can be the result of environmental variables, such as contest over food resources (Matsumura 1999), but may also be context-dependent (Funkhouser et al. 2018b). Grade one despotic species are generally marked by dominant individuals that show intense and highly asymmetrical patterns of aggression, little tolerance around resources, and infrequent reconciliation (e.g., M. mulatta, M. fuscata, and M. cyclopis). Species with a grade four dominance style show the opposite tendencies, with low or moderate levels of kin bias in affiliation, tolerant and supportive interactions with group members, strong group cohesion, and maternal tolerance for infant handling, for example, M. maura, M. nigra, M. ochreata, and M. tonkeana, all endemic to Sulawesi (Thierry et al. 2000). Although the above species fit easily onto this graded scale, other macaques are more difficult to categorize based on inconsistent or a lack of behavioral data. The classification of species as a grade two or three can often rely on relative comparisons: grade two macaques possess behavioral traits that are more similar to grade one species, and grade three macaques are more similar to grade four species.

Regardless of their place on this graded scale, the variation in social tolerance observed across the 23 extant macaque species provides an opportunity to directly compare different facets of behavior, including play structure and content, across multiple, differing social structures. Currently, much of the play research in macaques derives from species occupying opposite ends of the dominance scale, 
and the differences in observed play style, rate of play, and play signaling have been suggested to reflect this social dominance style (Petit et al. 2008; Reinhart et al. 2010; Yanagi and Berman 2014b). The social play of despotic species, such as rhesus (M. mulatta) or Japanese macaques (M. fuscata), can be characterized as competitive, whereas more tolerant species, such as Tonkean (M. tonkeana) or Sulawesi crested macaques (M. nigra), engage in a more cooperative play style (Petit et al. 2008; Reinhart et al. 2010). These differences are reflected in the targets attacked during play fighting as well as the type and frequency of play signals utilized throughout a play bout (Reinhart et al. 2010; Scopa and Palagi 2016; Yanagi and Berman 2014b). A more competitive or risky play style, one in which sensitive targets like the face are attacked, may generate miscommunication between partners and risk ending the play contact. Therefore, using play signals to indicate imminent play (Yanagi and Berman 2014a) or to reinforce playful intent (Wright et al. 2018) could be used to mitigate potential aggression and prolong the interaction (Scopa and Palagi 2016). These signals may be more specific and less interchangeable in more aggressive or competitive species to minimize the risk of misinterpretation (Scopa and Palagi 2016; Thierry et al. 2000; Yanagi and Berman 2014b) whereas in more tolerant species, these signals may be used redundantly and interchangeably to initiate/terminate play (Pellis et al. 2011; Scopa and Palagi 2016). Thus, investigating grade two and grade three macaque species, such as Tibetan macaques (M. thibetana), can help to determine the degree of overlap in species play patterns and play signaling.

\subsection{Tibetan Macaques}

Tibetan macaques are female philopatric and live in multi-male, multi-female social groups of 15-50 individuals (Berman et al. 2004; Thierry 2011; Thierry et al. 2000). This species has a strong kin bias with linear male and female dominance hierarchies, in which males generally occupy the top ranks of the hierarchy although females can outrank them (Berman et al. 2004). Despite being originally designated as having a grade three dominance style (Thierry et al. 2000), Tibetan macaques have been re-established as grade two, showing some despotism and low conciliatory tendencies, especially for female-female interactions (Berman et al. 2004). However, some female behavior is also inconsistent with a more despotic style, including that female individuals display a markedly high preference for female kin in proximity relationships and maternal tolerance for infant handling (Berman et al. 2004).

Female M. thibetana rank is based on matrilines with a daughter occupying the rank directly below her mother and above her older siblings (Berman et al. 2004; Thierry 2011; Zhao 1997). This hierarchy influences intergroup competition among females and generates preferential bonds between kin (Thierry 2011). Tibetan macaques groom at symmetric rates (exchange grooming for grooming received) and prefer female kin grooming partners, and females prefer to groom higher- 
ranking females (even if unrelated) (Xia et al. 2012). These investigations illuminate the generally despotic nature of Tibetan macaque social organization (Berman et al. 2004; Thierry et al. 2000), bias for female kin across a number of social contexts (viz., coalitionary support, grooming, and infant handling; e.g., Berman et al. 2004), and the overall value of grooming in this species (e.g., Xia et al. 2012, 2013). Tibetan macaques and other species with intermediate, graded behavioral variability may demonstrate behavioral nuances that are more likely a difference in degree rather than kind; therefore, it is important to determine the extent of these differences and how they manifest and compare across species.

Similar to other macaque species, Tibetan macaque juveniles engage in dyadic and polyadic play (Wright et al. 2018) that is fast-paced and rough and tumble. Few studies have examined social play in juvenile Tibetan macaques, primarily because few studies have been conducted on this species compared to other species of macaques. The play studies that have been performed have occurred at Valley of the Wild Monkeys in Anhui Province, Huangshan, China, specifically with the Yulingkeng A1 (YA1) group. Juveniles in this group have been noted as preferring similarly aged partners for both social play bouts and affiliation (Batts 2012). The type of play engaged in, whether solitary or social, also appears to depend on the age of the individual: infants tend to engage in more solitary play and juvenile males engage in the most social play (Batts 2012). The majority of juveniles in this group participate in play, but participation frequency, duration, and the rate of play signaling is variable (Wright et al. 2018). Nine candidate play signals, such as crouch and stare, have been observed, six of which overlap with the play signal repertoire of rhesus macaques (Yanagi and Berman 2014a). In this group, the play face is an important signal used throughout bouts but is not a reliable indicator that play will be initiated (see Wright et al. 2018 for a more detailed discussion). Having other individuals in proximity to the play bout (an audience) also seems to impact the play signaling of individuals involved, i.e., the data represent a negative parabolic trend. Play signaling in these juveniles increased as the number of audience members increased from zero to two but decreased with additional individuals beyond this threshold suggesting that the communication value of the play signal may degrade as the complexity and size of the play group increases (Wright et al. 2018). However, additional comparative research on this topic is necessary.

In the following example, we build upon previous social play studies of the YA1 juvenile Tibetan macaques of Mt. Huangshan, China, using social network analyses to supplement our current understanding of the social dynamics of this group. Although the majority of research performed on these macaques highlights the relationships between the adults, such analyses can help construct a more complete picture of group social dynamics. Additionally, this information can be used to generate future hypothesis-driven research about potential adult relationships and social position within the group as the juveniles age and become integrated into the adult social network. 


\subsection{Study Subjects and Data Collection}

The YA1 group of Tibetan macaques resides at Valley of the Wild Monkeys in the Huangshan Scenic District, Anhui Province, China. This group has been habituated to human presence since 1986 (see Berman and Li 2002; Berman et al. 2004) and is free-ranging but regularly provisioned with corn multiple times per day in supplement to their natural diet. All group members are individually recognized, and records of the group structure, including name, sex, birth date, and matriline, are maintained by Anhui University researchers. Additional adult female grooming data, collected July 7-August 28, 2016, between 06:30 and 17:30, was used to supplement some of the following analyses. To generate maternal dominance rank, all-occurrence sampling (Altmann 1974) was used to collect agonistic data from July 14 to August 27, 2016, from 7:00 to 12:00 and 14:00 to 17:00 daily (data contributed by Lori K. Sheeran). All research herein was approved by the Central Washington University Institutional Animal Care and Use Committee (protocols: \#A051602, \#M061603, \#A051702), and all protocols adhered to the legal requirements of the People's Republic of China and the American Society of Primatologists' Principles for the Ethical Treatment of Primates.

\subsubsection{Maternal Allogrooming and Dominance Rank}

In summer 2016, the YA1 group was composed of 47 individuals (19 males, 28 females): eight adult males, 13 adult females, and 26 infants and juveniles (between the ages of approximately 30 days and 6 years old) (see Table 6.1). All adult females were randomly sampled, and 10-min focal follows were conducted to collect all instances of auto- and allogrooming. This generated approximately 400 min of observation time per focal individual. Data were collected on actor/ recipient identities, rank, sex, matriline, and duration. An allogrooming bout was initiated with physical touch between partners, and the bout ended when all grooming partners ceased to groom for $>10$ s. If a grooming bout was polyadic (more than two individuals), the identity and durations of all partners were recorded, and the interactions were treated as dyadic.

To determine maternal rank, agonistic data were collected. Agonism consisted of fear grin, scream, flee, displace, threat, lunge, chase, grab, slap, and bite (as defined by Berman et al. 2004). We coded unambiguously directed agonistic interactions (or "competitions") in a 1:0 dichotomous fashion, where 1 indicated the "actor" who "won" the interaction and 0 indicated the "recipient" who "lost" the interaction. For these reasons, submissive behaviors (lack of agonism) were reverse-coded, where the actor was said to have lost (0) to the winning (1) recipient. We then derived Elo scores for each individual using methods similar to Neumann et al. (2011) in R. 
Table 6.1 Yulingkeng A1 player demographics in 2017

\begin{tabular}{|c|c|c|c|c|}
\hline Name & Age (days) & Age (years) & Sex & Mother \\
\hline TouQiuGuo (TQG) & 30 & $<1$ & $\mathrm{~F}$ & TouXiaHua \\
\hline YeXiaYun (YXYun) & 49 & $<1$ & $\mathrm{~F}$ & YeHong \\
\hline YeXiaDuo (YXD) & 65 & $<1$ & $\mathrm{~F}$ & YeChunYu \\
\hline YeXiaMing (YXM) & 102 & $<1$ & M & YeChunLan \\
\hline HuaXiaYun (HXYun) & 126 & $<1$ & $\mathrm{~F}$ & HuaHong \\
\hline TouHuaLi (THL) & 399 & 1 & $\mathrm{~F}$ & TouRongYu \\
\hline TouFuHua (TFH) & 451 & 1 & $\mathrm{~F}$ & TouHuaYu \\
\hline TouQiuYing (TQY) & 474 & 1 & $\mathrm{~F}$ & TouXiaXue \\
\hline YeXiaYue (YXYue) & 794 & 2 & $\mathrm{~F}$ & YeHong \\
\hline TouQiuSong (TQS) & 802 & 2 & M & TouXiaHua \\
\hline TouHuaNan (THN) & 823 & 2 & M & TouRui \\
\hline HuaXiaYue (HXYue) & 852 & 2 & $\mathrm{~F}$ & HuaHong \\
\hline YeChunHua (YCH) & 1461 & 4 & F & Unknown \\
\hline YeRongLan (YRL) & 1514 & 4 & $\mathrm{~F}$ & YeZhen \\
\hline TouRongXi (TRX) & 1537 & 4 & $\mathrm{~F}$ & TouTai \\
\hline TouQiuLan (TQL) & 1549 & 4 & $\mathrm{~F}$ & TouXiaXue \\
\hline HuaXiaWei (HXW) & 1579 & 4 & F & HuaHong \\
\hline YeXiaKun (YXK) & 1588 & 4 & M & YeHong \\
\hline HuangYu (HY) & 1643 & 5 & M & Unknown \\
\hline YeChunLan (YCL) ${ }^{\mathrm{a}}$ & 1740 & 5 & $\mathrm{~F}$ & YeMai \\
\hline TouXiaLong (TXL) & 1817 & 5 & M & TouHong \\
\hline TouHuaXue (THX) $)^{a, b}$ & 1889 & 5 & $\mathrm{~F}$ & TouRui \\
\hline TouRongYu (TRY2) & 1969 & 5 & M & TouTai \\
\hline YeChunLong (YCLong), & 2557 & 7 & M & YeMai \\
\hline YeRongQiang (YRQ) $^{\mathrm{a}}$ & 2586 & 7 & M & YeZhen \\
\hline TouRongGong (TRG) ${ }^{\mathrm{a}}$ & 2635 & 7 & M & TouTai \\
\hline HuaXiaMing $(\mathrm{HXM})^{\mathrm{a}}$ & 2675 & 7 & M & HuaHong \\
\hline
\end{tabular}

Age was calculated from the recorded birth date to 6-21-2017, which was when Anhui University researchers prepared the demographic information for the field season. In instances where the birth date and matriline were unknown (e.g., YeChunHua and HuangYu), age was estimated based on the individual's size and sex characteristics

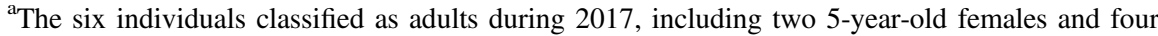
7-year-old males

${ }^{\mathrm{b}}$ The two adult individuals of the six who participated in play with younger individuals (THX and YCLong)

\subsubsection{Juvenile Play Behavior}

In summer 2017, the YA1 group was composed of 46 individuals (17 males, 29 females): 10 adult males, 15 adult females, and 21 infants and juveniles (between the ages of approximately 30 days and 5 years old). Data were collected from July 6 to August 5, 2017, between 06:30 and 17:30. A complete list of player demographics, including age, sex, and matriline, are shown in Table 6.1. 
Video footage of social play behavior was collected using all-occurrence sampling and a Sony Handycam camcorder. Regular scans of infant and juvenile interactions occurred until social play was initiated. If a second play bout occurred while recording the first, the video frame was widened to include both bouts. If this was not possible, the first bout was recorded to completion, and then the second bout was recorded. The juveniles were followed until play concluded and the players ceased to engage in play for $>10 \mathrm{~s}$. All play videos were coded using VLC media player (version 3.0.3, Vetinari) and Microsoft Excel (version 16.14) for player identity, demographic information (e.g., age, sex, and matriline), duration of the play bout, and the total number of players involved. All ages were recorded in days, and all durations were recorded in seconds.

A coding distinction was made between the longer encompassing play bout and the smaller component units designated here as play periods (see Mayhew 2013 for a discussion of play structure, including play periods and vigilance periods). Here, only play periods were examined. Guided by Burghardt (2005) and Fagen (1981), the start of a play bout was marked by the exchange of playful behavior between two or more juveniles. A play bout was considered to have concluded when (1) a player engaged in non-playful behavior (e.g., aggression), (2) a non-player directed non-playful behavior toward the players (e.g., the bout was interrupted by an adult initiating a grooming session), or (3) player(s) withdrew from the interaction, thus dissolving the play group. Because play dynamics change quickly, it was noted each time a player withdrew from or was added to the interaction even if play continued following this change in player composition. Each partner interchange marked the transition to a new play period; for example, players A and B are playing, and player $\mathrm{C}$ approaches and joins; therefore the play period between $\mathrm{A}$ and $\mathrm{B}$ terminates and a new period containing players $\mathrm{A}, \mathrm{B}$, and $\mathrm{C}$ begins (see example in Fig. 6.1).

\subsubsection{Statistical Analyses}

As indicated by its frequent appearance in recent animal behavior studies, social network analysis (SNA) has become a valuable tool for understanding the role and

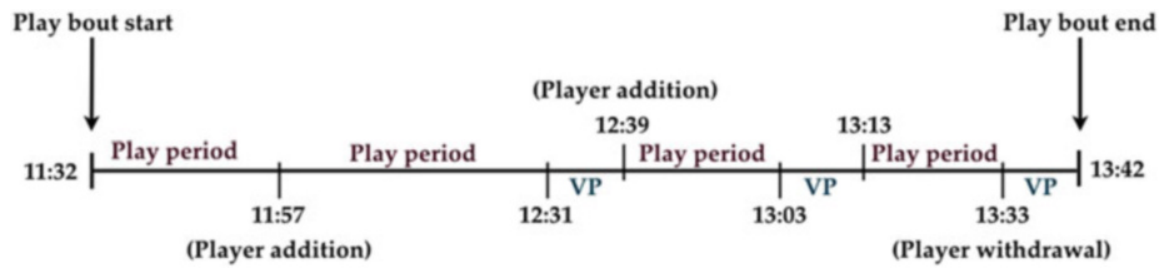

Fig. 6.1 An example of a play bout divided into its smaller components: play periods and vigilance periods (VP). A change in player composition (addition or withdrawal) is marked and indicates a transition into a new play period (adapted from Mayhew 2013) 
positioning of individuals within a larger social group. SNA uses matrix-based data to analyze individual social interactions, where individuals can be depicted as nodes and network-based descriptive and statistical analyses are generated to describe the relationship between nodes (Sueur et al. 2011a; Whitehead 2008). A comprehensive overview of the utility of SNA, its terminology, methods, and analyses can be found elsewhere in comprehensive texts (see Borgatti et al. 2013 or Whitehead 2008).

SNA has been used to characterize these relationships in primate social groups, as well as identify clusters of individuals, subgroups, isolated group members, and, overall, diagram of the group's social network (e.g., Clark 2011; Farine and Whitehead 2015; Funkhouser et al. 2018a; Sueur et al. 2011a). For example, using SNA, researchers have examined the correlation between juvenile play network positions, ontogenetic social development, and later-life social connectedness (P. troglodytes, Shimada and Sueur 2014; M. fuscata, Shimada and Sueur 2017; Macaca spp., Sueur et al. 2011b). Animals that are strongly connected to one another (i.e., having strong bonds) tend to have relationships on multiple independent measures of relation; thus, it can be expected that playing preferentially with certain partners will correlate with other measures, such as grooming, copulation, and proximity (Whitehead 2008).

In this study, all statistical analyses were performed using IBM SPSS Statistics (Version 23) software ( $\alpha=0.05$ ). UCINET (Borgatti et al. 2002) was used to calculate network statistics, and NetDraw was used to construct sociograms. Elo scores (calculating position in the dominance hierarchy) were derived using R. To investigate correlations between independent social networks (e.g., durations of play and difference in age) we used QAP correlation analyses in UCINET; this test analyzes whole matrices against one another. The following node-by-node statistics were calculated: (1) degree (the sum of each node's ties with all other nodes, also known as strength), (2) eigenvector centrality (how well an individual is associated with others and how well the associates are associated), (3) closeness (how close nodes are within a network, often discussed in terms of the time it takes for information to spread from one individual to others), and (4) betweenness (a value used to assess a node's ability to control flow through a network). We also constructed a principal coordinate sociogram to illustrate the observed social play relationships between individuals. This sociogram plots individuals with strong associations near one another. To define the minimum edge value in this diagram, we used the mean of all directional dyadic indexes plus one standard deviation $($ mean + SD). Detailed information about these node-by-node statistics can be found in Borgatti et al. (2013) and Whitehead (2008).

\subsection{Results}

In total, 256 play bouts (12,458 s total) were observed containing 965 play periods. Twenty-three macaques (eight males, 15 females) participated in play, ranging in age from approximately 30 days (TQG) to 7 years old (YCLong) (Table 6.1). Play bouts ranged in duration from one to $895 \mathrm{~s}$ (nearly $15 \mathrm{~min}$ ), but the mean was 
considerably shorter $(48.99 \pm 80.33 \mathrm{~s})$. Play periods ranged in duration from 1 to $227 \mathrm{~s}$ with a mean of $12.91 \pm 16.70 \mathrm{~s}$.

\subsubsection{Player Age}

Play was observed more frequently between players of the same birth cohort (i.e., individuals born in the same year) (QAP, $r=0.289, p=0.001$ ) and age class (QAP, $r=0.219, p<0.001)$. The mean age of a player was $867.66 \pm 496.55$ days old (approximately 2.4 years old), and the mean age of male players $(977.75 \pm 488.22$ days) was slightly older than female players (736.84 \pm 476.56 days). A Pearson correlation was used to determine whether the age of the player (in days) correlated with the total amount of time spent playing with any other player(s) during the study period. Player age was significantly correlated with play duration $(r=-0.246, p \leq 0.001)$, and this negative relationship suggests that as age increased, the time spent involved in play decreased. Notably, when only dyadic play periods were examined, the ages of the players were positively correlated ( $r=0.467, p \leq 0.001)$, indicating that as the age of Player 1 increased, the age of Player 2 also increased (Fig. 6.2).

Play Partner Ages in Tibetan Macaque Dyadic Play Bouts

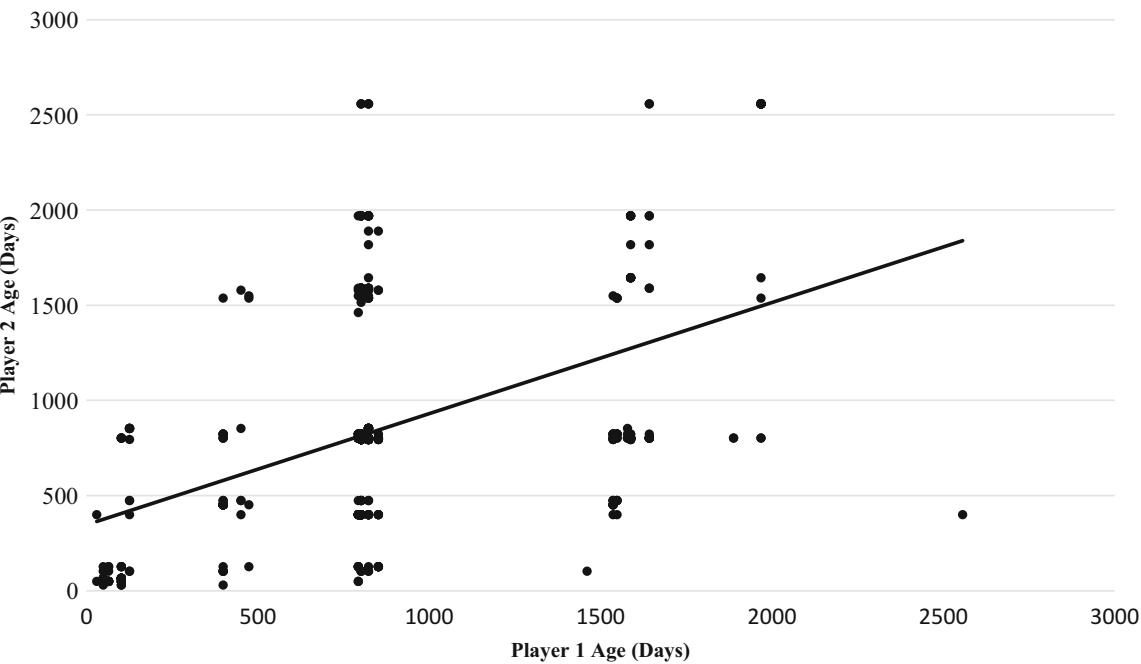

Fig. 6.2 A scatterplot of player ages in dyadic play bouts (two individuals) indicating that as the age of one partner increased, the age of the second partner also tended to increase $(r=0.467)$ 


\subsubsection{Number of Players}

Consistent with Wright et al. (2018), polyadic play periods were observed in this group of macaques (four players, $n=66$; five players, $n=10$ ) but occurred less frequently than dyadic $(n=628)$ or triadic $(n=261)$ interactions; the mean number of players in a play period was $2.44 \pm 0.67$. Similarly, the total number of players in a play period was significantly correlated with the duration of a period $(r=-0.138$, $p \leq 0.001$ ), indicating that playing with more individuals decreased the duration of play. Additionally, the total number of players in a play period was significantly correlated with player age, indicating that as player age increased, the number of players involved decreased $(r=-0.152, p \leq 0.001)$.

\subsubsection{Player Composition}

An analysis of player composition indicated that mixed sex play ( $n=533$ periods, $55.2 \%$ ) represented more than half of all play periods (all-male play, $n=273$, $28.3 \%$; all-female play, $n=159,16.5 \%$ ), and this was significantly different from the expected values $\left(\chi^{2}(2)=228.47, p \leq 0.001\right)$. Therefore, juvenile males remained active participants despite the presence of more juvenile females in the group.

\subsubsection{Matrilineal Relatedness and Rank}

YA1 matrilines are known and documented annually by Anhui University researchers; therefore it was possible to determine and rank the degree of maternal relatedness between juveniles. There was no significant relationship between maternal difference in Elo score (dominance) and the duration of play in offspring (QAP, $r=0.00, p=0.025)$. Similarly, there was no significant relationship between maternal dominance status (dominant or subordinate) and the duration of play in offspring (QAP, $r=-0.025, p=0.411$ ).

\subsubsection{Maternal Social Relationships}

Adult female grooming duration data from 2016 was factored in as a proxy for maternal social dyadic relationships, but there was no significant relationship between maternal grooming (total seconds) and the duration of play in offspring (QAP, $r=0.018, p=0.274$ ). 


\subsubsection{Individual Playfulness}

In addition to the above demographic variables, individualistic patterns emerged for the juveniles in this group. Predictably, some juveniles played more frequently and longer than others (see Figs. 6.3 and 6.4), and overall, TouQiuSong (TQS), TouHuaNan (THN), and YeXiaYue (YXYue) were the three most active participants (appearing in 56.58, 40.70, and $32.40 \%$ of play periods observed, respectively). All three of these players were born within 29 days of one another and belonged to the 2015 birth cohort. Interestingly, the oldest individual in this cohort, HuaXiaYue (HXYue), was born 29 days before THN, but did not participate in play nearly as much as her three peers.

Network statistics for each player were calculated, including degree, eigenvector centrality, closeness, and betweenness (Table 6.2). To better visualize these play relationships, a sociogram was constructed (minimum edge weights of mean \pm SD) for all dyadic interactions (Fig. 6.5). Examining the network statistics and sociogram, TQS, THN, and YXYue appear to unite three clusters of players.

Based on a cursory examination of the play frequency and duration data as well as from personal observation, it is unsurprising that TQS, THN, and YXYue were the

\section{Individual Macaque Play Participation Frequencies}

600

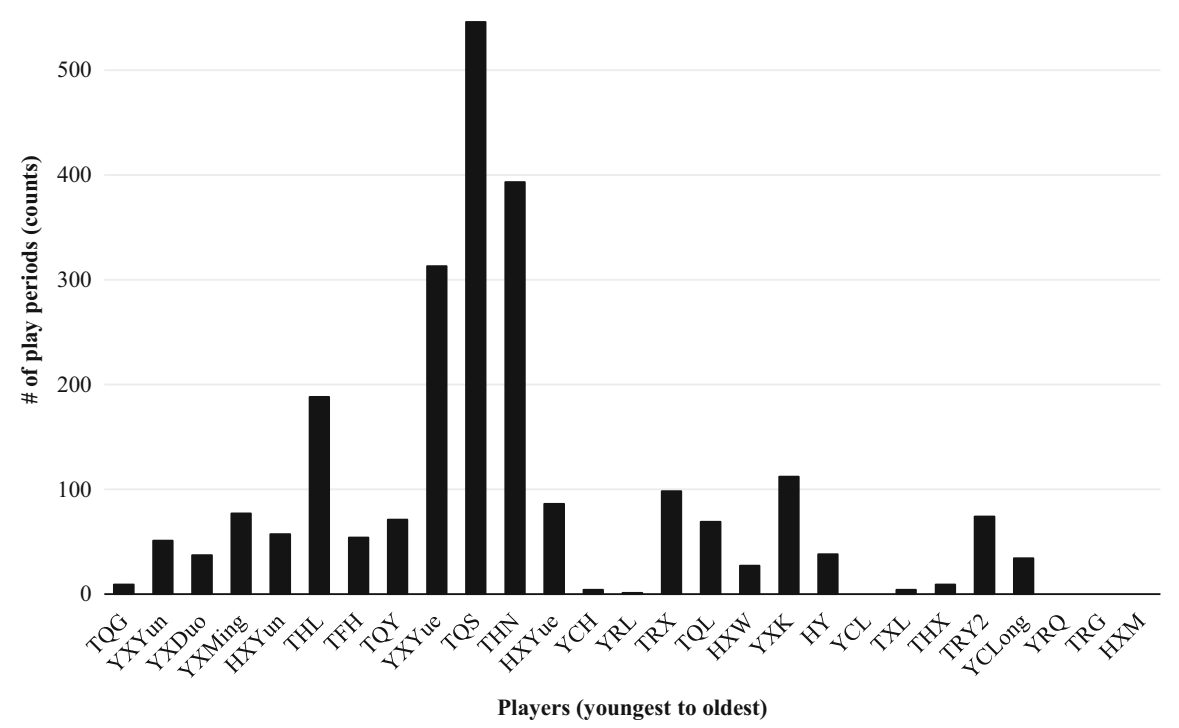

Fig. 6.3 The total individual counts of play participation for all group members $\leq 7$ years old in the YA1 group during 2017. TouQiuGuo (TQG) on the left is the youngest player at 30 days old and HuaXiaMing (HXM) is the oldest at 2675 days. Some individuals were not observed to engage in play at all (e.g., HuaXiaMing), whereas others (e.g., YXYue, TQS, and THN) were frequent participants 


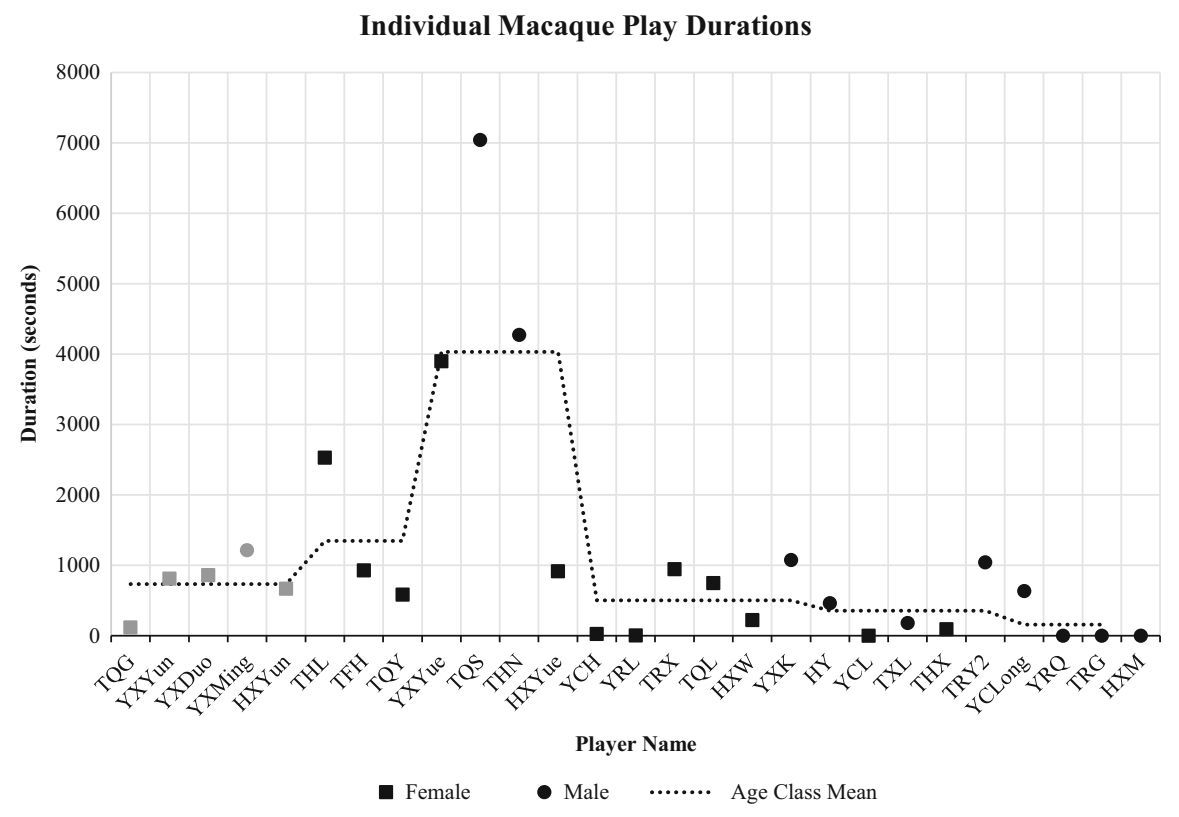

Fig. 6.4 The total individual durations of play (in seconds) for all male and female individuals $\leq 7$ years old. Males are depicted as circles, females as squares, infants as gray, and juveniles as black. Individuals are organized left to right by age in days (TQG is the youngest, HXM is the oldest). The dotted line represents the mean play duration for each age class, including all individuals of that age class even if they were not observed playing (e.g., YCL, YRQ, TRG, and HXM)

top three ranking individuals for all four calculated network measures. Overall, TQS has the highest strength (6896) and betweenness (47.905) values, indicating that he is highly gregarious and links discrete clusters of juvenile players. His high eigenvector centrality of 1.0 indicates that he is well connected to others that are themselves well connected within the network. He also has the lowest closeness value (24), which suggests that social information that originates at a random node in the network would reach him quickly and with high fidelity, again emphasizing his central position in the network. The results are very similar for THN and YXYue, and these three juveniles are found clustered in the center of the sociogram.

The individuals in the three clusters evident in Fig. 6.5 are notably comprised of individuals with similar demographic characteristics. Cluster A contains the group's older and larger males (ages four to seven), including YeChunLong (YCLong), TouRongYu (TRY2), HuangYu (HY), and YeXiaKun (YXK). Cluster B contains nearly all remaining juveniles (ages two to four), who are predominantly female, including TouRongXi (TRX), TouQiuLan (TQL), TouQiuYing (TQY), TouHuaLi (THL), and TouFuHua (TFH). Cluster C contains HXYue (age three) and the majority of the group's infants: YeXiaYun (YXY), YeXiaDuo (YXD), and YeXiaMing (YXM). Clusters $\mathrm{A}$ and $\mathrm{C}$ appear to be connected via cluster B, 
Table 6.2 2017 Yulingkeng A1 player network measures sorted by degree

\begin{tabular}{l|l|l|l|l}
\hline Player & Degree & Eigenvector centrality & Closeness & Betweenness \\
\hline TQS & $\mathbf{6 8 9 6}$ & $\mathbf{1}$ & $\mathbf{2 4}$ & $\mathbf{4 7 . 9 0 5}$ \\
\hline THN & $\mathbf{4 4 4 8}$ & $\mathbf{0 . 7 4}$ & $\mathbf{2 6}$ & $\mathbf{2 8 . 6}$ \\
\hline YXYue & $\mathbf{4 1 0 8}$ & $\mathbf{0 . 7 9 1}$ & $\mathbf{2 5}$ & $\mathbf{2 8 . 8 0 7}$ \\
\hline THL & 3393 & 0.579 & 30 & 6.211 \\
\hline YXMing & 1351 & 0.062 & 30 & 13.186 \\
\hline TRX & 1266 & 0.153 & 34 & 1.5 \\
\hline TQL & 1237 & 0.145 & 35 & 0.25 \\
\hline YXK & 1215 & 0.216 & 37 & 1.715 \\
\hline TRY2 & 1201 & 0.15 & 36 & 1.394 \\
\hline TQY & 1187 & 0.155 & 32 & 1.313 \\
\hline YXDuo & 1027 & 0.016 & 46 & 0.2 \\
\hline YXYun & 1023 & 0.054 & 35 & 3.781 \\
\hline TFH & 1005 & 0.124 & 32 & 1.153 \\
\hline HXYue & 879 & 0.144 & 32 & 1.416 \\
\hline HXYun & 722 & 0.07 & 32 & 7.197 \\
\hline YCLong & 719 & 0.056 & 39 & 0.966 \\
\hline HY & 539 & 0.088 & 36 & 2.276 \\
\hline HXW & 291 & 0.045 & 34 & 0.862 \\
\hline TXL & 188 & 0.014 & 43 & 0.2 \\
\hline TQG & 161 & 0.006 & 40 & 1.069 \\
\hline YCH & 36 & 0.008 & 40 & 0 \\
\hline YRL & 4 & 0.001 & 44 & 0 \\
\hline B & & &
\end{tabular}

Bold indicates the top three individuals with the highest network measures

suggesting that certain individuals or individuals of intermediate age more generally act as scaffolding for infants into the juvenile social network. One juvenile was a notable social isolate with the lowest degree, eigenvector, and betweenness values and highest closeness value: YeRongLan (YRL), who only participated in one play bout for four seconds. Similarly, other individuals had few or weak social network connections, reflected by their low degree, eigenvector, and betweenness values and high closeness scores: YeChunHua (YCH), TouQiuGuo (TQG), TouXiaLong (TXL), and HuaXiaWei (HXW). These isolated individuals were both males and females and ranged from infancy to nearly 5 years old.

\subsection{Discussion}

The adult macaques in the YA1 group are well known and studied, but juvenile interactions have not been well documented. The results outlined in this chapter provide a window into the social lives of these juveniles, and the results presented here build upon previous Tibetan macaque social play research performed at this site (viz., Wright et al. 2018). Importantly, the findings in this work aim to establish these 


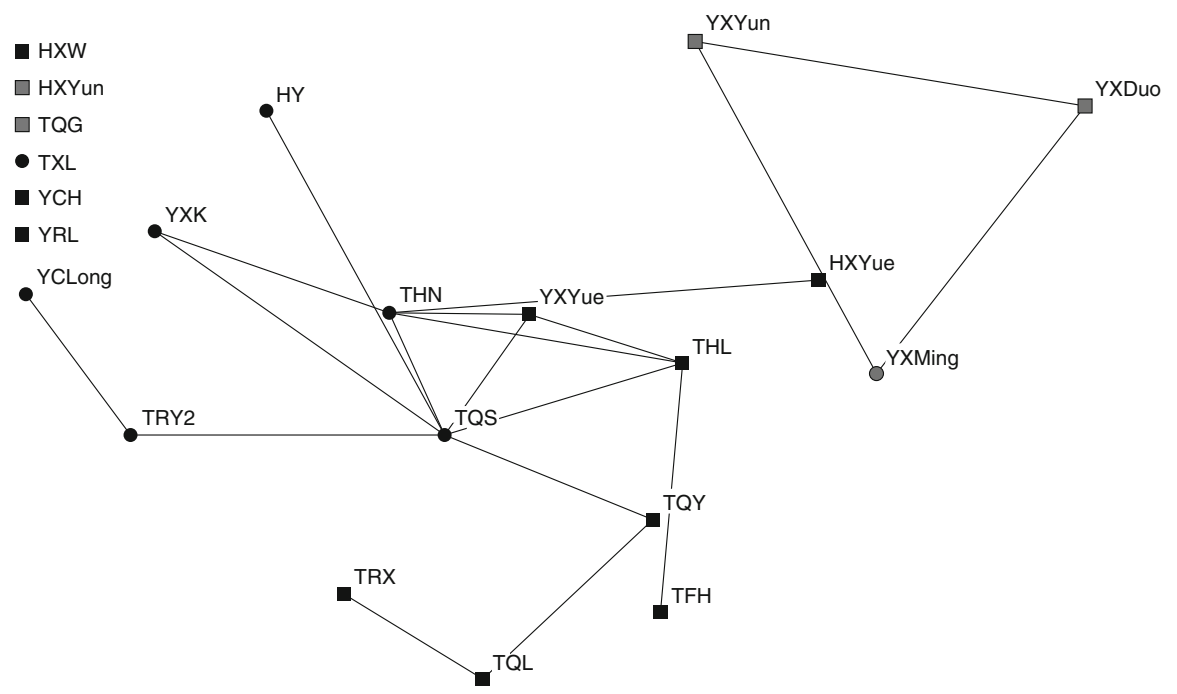

Fig. 6.5 Sociogram for the 2017 juvenile and infant play network based on duration data (mean $\pm \mathrm{SD}=242$ ). This network highlights those juveniles who participated more frequently in social play. Males are depicted as circles, females as squares, juveniles as black, and infants $(<1$ years old) as gray. YCLong is included in this network as a juvenile but was categorized as an adult at the time of the study

juveniles as important entities within the group that have their own identities and the potential to drive the direction of group social dynamics as they age and continue to interact.

The frequency of social play in these juvenile macaques followed a similar trajectory to other primate species: play increased as age increased, peaked at approximately 2.5 years old, and then declined. Adult macaques in this group rarely participated in play, which supports previous literature indicating a general lack of adult primate play in more despotic species. Additionally, the number of players involved in a play period was fewer when the participating players were older. The QAP results indicated that juveniles had a preference for certain social partners and played more frequently with peers of their birth cohort. In dyadic play bouts, partner ages were positively correlated, which is a further indication of a preference for similarly aged partners. Taken together, partner preferences may not only be indicative of the strong social bonds that result from growing up together in the same age cohort but also reflect a preference for equity in social competition (Kulik et al. 2015; van Leeuwen et al. 2011). As juveniles age, especially males, physical strength and prowess build and are increasingly put to the test in competition with other individuals. This partner equity may be particularly important for more despotic macaque species in attempt to ensure that play occurs with an appropriately matched opponent in skills, abilities, and size in attempt to reduce the risk of play devolving into aggression by interacting with a mismatched partner. Tibetan macaques also engage in polyadic play, which can quickly become complicated for the participants as well 
as the researchers observing the interaction. Typically, the duration of these polyadic play events is shorter than when play occurs between dyads. This is reasonable considering that when more players become involved in an interaction, it becomes increasingly difficult for each player to monitor their own behavior as well as track the behaviors of all the other partners simultaneously. This inability to effectively monitor multiple partners may contribute to the brevity of polyadic play. Such events also open the door for future research analyzing the structure and composition of these bouts in comparison to dyadic events.

Although there may be some general conclusions to be made about play in macaques depending on their degree of social despotism or tolerance, the influence of individual identity and personality on play participation warrants further exploration. Some individuals, simply based on personality traits (e.g., boldness, tendency to explore, or sociability), may be more inclined to participate in play regardless of their partner's age, sex, or relationship. Although personality data were not collected for these juvenile macaques, and the dataset here only represents a snapshot of the relationships of this group, predictions could be made about which juveniles likely possess which traits and to what degree. This is undoubtedly a large undertaking; however, such data could generate some understanding of how personality contributes to social play participation and can aid in generating predictions about how certain individuals will behave in other realms of social behavior.

\subsubsection{Does Social Position Matter?}

The introduction of SNA into social play analyses is useful in that it not only literally illustrates the complex relationships shared by juveniles but also provides a method to track individual relationships and compare them across years, events, and other social networks (e.g., Shimada and Sueur 2017). Specifically, SNA allows researchers to follow an individual across time and various social networks to investigate the effects of an individual's characteristics during play as a juvenile (e.g., frequency, partner choice and diversity, and play style) on their position in adult social networks. Such a longitudinal, multilayer social network framework would provide a pointed investigation of the adaptive mechanisms present during the juvenile play period that may impact reproductive fitness later in life (e.g., dominance status, reproductive success, infant survival). The incorporation of such information into future social play studies may be of interest to researchers performing long-term studies, where data-driven predictions about the role or tenure of future juveniles within the social group is invaluable. For the YA1 group, this type of information may be helpful to future researchers interested in the development and maintenance of Tibetan macaque relationships.

In 2017, it appears that TQS, THN, and YXYue provided the foundation for this social play network in 2017, acting as social glue and connecting juveniles across multiple birth cohorts and matrilines. Interestingly, the grandmothers of THN and TQS were central in the 2016 female allogrooming network (TouTai and TouHong, 
respectively, unpublished data), so it is possible that well-connected maternal relatives influence the social freedom or social position of kin across years. Whether the central positioning of this trio is an artifact of their particular age (nearly 3 years old), their early cohort experience (close dates of birth), or previous sibling or kin scaffolding into the social network is currently unknown, but additional SNA data from 2015 and 2016 can help to clarify this picture.

The central nature of TQS, THN, and YXYue can also be used to make predictions about the transition of future dominance status and mating opportunities in this group. The YA1 group has a history of some resident males remaining in the group longer than expected and rising through the ranks to alpha position (e.g., YeRongBing, HuaXiaMing, and TouGui). This longer group tenure might also be expected of THN and TQS, and they may not be immediately pressured by group males to disperse. As a female, YXYue is expected to remain in YA1, and once she reaches sexual maturity, she will likely fall into place at the top of the hierarchy with the rest of her Ye female relatives, achieving this high rank namely because her mother, YeHong, is the alpha female.

Social predictions can also be made about individuals that occupy more peripheral positions in the juvenile network. For some, such as the infants of the group, this position will most likely change with age and increased social exposure to their peers or may be mediated by the infant's matriline. For others, this window of social opportunity may have closed, and this peripheral social position may persist into adulthood. One important advantage of reliably identifying unique infants and juveniles is that it allows for young individuals to be tracked across development to test for changes in the status of their social lives. Further, reliably identifying young individuals ensures control for bias in data collection, whereby ensuring that nongregarious or cryptic individuals (that might be easily overlooked because they play less) can be accurately represented in a dataset.

Although the infants in this group all had low eigenvector values (less central), they comprised their own cluster and the majority were not social isolates (peripheral). The isolation of TouQiuGuo (TQG) from the juvenile network can be explained by her very young age (a newborn). The clustering of the remaining newborn peer group may have occurred because of limited social access and ability. For infants, having access to social partners beyond their mother can be restricted because macaque mothers often carefully control their offspring's experience during their first vulnerable months. For Tibetan macaques, this can be a physical restriction, where the mother will not release the infant from her grasp, or a social restriction, where the mother actively monitors and interferes in the social interactions between her offspring and other group members for at least the initial weeks of life. Indeed, Tibetan macaque mothers often affiliate or associate with one another, thereby providing limited opportunities for their infants to socially engage while still being heavily monitored. Regardless, this mothering behavior limits the infant's ability to socially engage relative to the rate of older (juvenile) conspecifics. Aside from the influence of maternal rearing behavior, the infant itself has limited physical abilities that prohibit it from keeping up with older players. Growth and development bring physical, mental, and emotional strength, flexibility, endurance, and 
knowledge of the natural environment, but this is inherently a slow, prolonged process, one that requires time as well as practice and repeated experience. A newborn infant is also a novel object in the environment that others must familiarize themselves with; therefore, it is not simply that the infant must gain physical and social competence to enter into this new social world; they must also gain acceptance from members of the social group and be treated accordingly by older play partners.

A handful of juveniles were social isolates. Both YCH and YRL were rarely seen interacting with any members of the YA1 group, juvenile or otherwise. $\mathrm{YCH}$ is thought to be a juvenile immigrant (rare), and therefore she lacks kin relations on which to base her social behaviors; this could explain her peripheral social position. YRL's mother, YeZhen, occupied the lowest position in the social hierarchy during 2017, and this low rank could have impacted YRL's social position within the juvenile network by limiting her opportunities for social engagement. It was surprising to uncover the weak connection of TouXiaLong (TXL) to the juvenile play network because his mother, TouHong, occupied a central, well-connected position within the group's 2016 grooming network (unpublished data). Further, TXL's maternal cousin is TQS, a highly playful and central individual (see above). Although the position of TXL was wholly different from TQS, a possible explanation for his position revolves around his age: TXL was roughly 5 years old and possibly in a liminal developmental stage where he was transitioning out of the juvenile period and therefore more concerned with establishing connections to the adult social network.

\subsubsection{Future Considerations}

One major challenge to studying social play in primates is that there are typically a large number of participants to track. When studying a large group, especially one containing many young individuals who look quite similar, it is considerably easier to use broad age categories to ease any comparisons (e.g., infant, juvenile, subadult, and adult). However, this generalization of individuals into broad age classes can generate statistical and conceptual issues because the boundaries between age categories differ widely between sexes, species, and studies. In primates, the majority of individuals involved in play are juveniles, which often means they are between the ages of weaning and sexual maturity. This, however, is a large and variable span of developmental time, and the acquisition of physical, social, and cognitive skills may occur at different stages and paces. Not only might there be marked differences in the physical and social abilities among the age classes, there are considerable differences in abilities within age classes, for example, between a 2-year-old juvenile who is 731 days old and a 2-year-old juvenile who is 1094 days old. Therefore, it is our recommendation to use age in days to achieve a fine-grain perspective and to emphasize the individual nature and developmental trajectory of play in any group examined. 
Much like any study on social play, more questions emerge and remain open for future exploration than are typically answered. In particular, the idea of social scaffolding - acting as a bridge and supporting or facilitating a connection between groups of individuals - is of particular interest. Who are the individuals that fall into this particular role? Are they kin? Do older siblings help assimilate younger siblings into the network? What is the influence of having a small familial network to begin with? These kinds of questions require longitudinal comparisons, that not only incorporate social play data but also multiple independent measures of association (Shimada and Sueur 2017), such as proximity and grooming.

Furthermore, questions of evolutionary importance surface in the study of social play. Particularly, because play is often regarded as a context for learning and practice for later serious adult interactions (Lutz et al. 2019), juvenile play is rich in opportunities to study the contributions of juvenile behavior to adult reproductive success. Do individuals who play at high frequencies when young go on to be more successful in dominance and mating interactions (relative to juveniles who rarely play)? What about those that demonstrate high play partner diversity? What is the effect of maternal or paternal social network connectedness on the juvenile's frequency or diversity of play? Do observed rates of play in different contexts (e.g., social or object) predict an individual's success in corresponding contexts later in life (e.g., mating or extractive foraging)? Researchers could test such predictions using unidimensional social network analyses (as we illustrate here) or expand on multidimensional social networks. Multiplex social network analyses are particularly useful in studying complex social systems where individuals participate in various social contexts (Smith-Aguilar et al. 2018). With multiplex statistics, researchers could ask questions of variation across multiple behaviorally defined layers (e.g., play, grooming, agonism, and mating), temporal sequences (e.g., years or developmental periods), or biologically relevant connections (e.g., kinship) to test predictions of inclusive fitness and reproductive success.

Studying play could also help to place the four grades of macaque species into clearer context: the differences between these species, especially those considered to be grades two and three, most likely reflect a nuanced degree rather than overt differences. Tibetan macaques are considered a grade two species, meaning that observers should see some evidence of despotism, hierarchical behavior, and matrilineal preference, even in juvenile play. However, researchers should expect to observe some behavioral inconsistencies when compared with grade one macaques (e.g., M. mulatta or M. fuscata). For example, contrary to the expectation for more despotic species, social play in this group is not often interrupted by an adult; instead, juveniles are fairly self-sufficient at self-regulating play bouts and choose when to participate rather than being controlled by the adults of the group (Wright et al. 2018). Additionally, neither kinship nor maternal rank appears to have a significant impact on the duration of play or partner choice (this study). These are unexpected results that warrant further inquiry and require researchers to ask themselves: just how much variation is reasonable to expect for grade two or three species? 
Acknowledgments We are so grateful for the continued support and guidance of Dr. Lori K. Sheeran. Without your collaboration, this work would not be possible. Many thanks to Dr. Crickette Sanz for stimulating discussions on the utility of social networks in an evolutionary context. We are excited for the future research realized from these conversations. We are also appreciative of our collaborators at Anhui University and the helpful and positive feedback provided by two reviewers of this piece. Finally, to all of the individuals of the YA1 group, thank you for letting us spend some time in your world.

\section{References}

Altmann J (1974) Observational study of behavior: sampling methods. Behav 49(3/4):227-267

Baldwin JD, Baldwin JI (1974) Exploration and social play in squirrel monkeys (Saimiri). Am Zool 14(1):303-315

Batts C (2012) The impact of eco-tourism on infant and juvenile play behaviors in Tibetan macaques (Macaca thibetana). Thesis, Central Washington University, Ellensburg, WA

Bekoff M (1972) The development of social interaction, play and metacommunication in mammals: an ethological perspective. Q Rev Biol 47(4):412-434

Bekoff M (1974) Social play and play-soliciting by infant canids. Am Zool 14:323-340

Bekoff M, Allen C (1997) Intentional communication and social play: how and why animals negotiate and agree to play. In: Bekoff M, Byers JA (eds) Animal play: evolutionary, comparative, and ecological perspectives. Cambridge University Press, New York, pp 97-114

Bekoff M, Byers JA (1981) A critical reanalysis of the ontogeny of mammalian social and locomotor play, an ethological hornet's nest. In: Immelmann K, Barlow GW, Petrinovich L, Main M (eds) Behavioral development, the bielefeld interdisciplinary project. Cambridge University Press, New York, pp 296-337

Berman CM, Li JH (2002) The impact of translocation, provisioning and range restriction on a group of Macaca thibetana. Int J Primatol 23(2):383-397

Berman CM, Ionica CS, Li JH (2004) Dominance style among Macaca thibetana on Mt. Huangshan, China. Int J Primatol 25(6):1283-1312

Borgatti SP, Everett MG, Freeman LC (2002) UCINET for Windows: software for social network analysis. Version 6.627 [software]. 2016 Dec 14. Available from https://sites.google.com/site/ ucinetsoftware/home

Borgatti SP, Everett MG, Johnson JC (2013) Analyzing social networks. Sage, Los Angeles, CA

Brown SG (1988) Play behaviour in lowland gorillas: Age differences, sex differences, and possible functions. Primates 29(2):219-228

Burghardt G (2005) The genesis of animal play: Testing the limits. MIT Press, Ann Arbor, MI

Burghardt G, Ward B, Rosscoe R (1996) Problem of reptile play: Environmental enrichment and play behavior in a captive Nile soft-shelled turtle, Trionyx triunguis. Zoo Biol 15:223-238

Burghardt G, Dinets V, Murphy JB (2014) Highly repetitive object play in a cichlid fish (Tropheus duboisi). Ethology 121:38-44

Byers JA, Walker C (1995) Refining the motor training hypothesis for the evolution of play. Am Nat 146(1):25-40

Ciani F, Dall'Olio S, Stanyon R et al (2012) Social tolerance and adult play in macaque societies: a comparison with different human cultures. Anim Behav 84:1313-1322

Clark FE (2011) Great ape cognition and captive care: Can cognitive challenges enhance wellbeing? Appl Anim Behav Sci 135:1-12

Cloutier S, Baker C, Wahl K et al (2013) Playful handling as social enrichment for individually- and group-housed laboratory rats. Appl Anim Behav Sci 143:85-95

Fagen R (1981) Animal play behavior. Oxford University Press, New York

Fagen R (1984) Play and behavioural flexibility. In: Smith PK (ed) Play in animals and humans. Basil Blackwell, Oxford, pp 159-173 
Farine DR, Whitehead H (2015) Constructing, conducting and interpreting animal social network analysis. J Anim Ecol 84:1144-1163

Fontaine RP (1994) Play as physical flexibility training in five ceboid primates. J Comp Psychol 108 (3):203-212

Funkhouser JA, Mayhew JA, Mulcahy JB (2018a) Social network and dominance hierarchy analyses at chimpanzee sanctuary northwest. PLoS One 13(2):e0191898

Funkhouser JA, Mayhew JA, Sheeran LK, Mulcahy JB, Li JH (2018b) Comparative investigations of social context-dependent dominance in captive chimpanzees (Pan troglodytes) and wild Tibetan macaques (Macaca thibetana). Sci Rep 8(1):e13909

Gamble JR, Cristol DA (2002) Drop-catch behaviour is play in herring gulls, Larus argentatus. Anim Behav 63:339-345

Gómez JC, Martín-Andrade B (2002) Possible precursors of pretend play in nonpretend actions of captive gorillas (Gorilla gorilla). In: Mitchell R (ed) Pretending and imagination in animals and children. Cambridge University Press, Cambridge, pp 255-268

Heinrich B, Smolker R (1998) Play in common ravens (Corvus corax). In: Bekoff M, Byers JA (eds) Animal play: evolutionary, comparative, and ecological perspectives. Cambridge University Press, New York, pp 27-44

Hill HM, Dietrich S, Cappiello B (2017) Learning to play: A review and theoretical investigation of the developmental mechanisms and functions of cetacean play. Learn Behav 45(4):335-354

Jones BL, Kuczaj SA (2014) Beluga (Delphinapterus leucas) novel bubble helix play behavior. Anim Behav Cogn 1(2):206-214

Kahlenberg SM, Wrangham RW (2010) Sex differences in chimpanzees' use of sticks as play objects resemble those of children. Curr Biol 20(24):R1067-R1068

Kulik L, Amici F, Langos D et al (2015) Sex differences in the development of social relationships in rhesus macaques (Macaca mulatta). Int J Primatol 36:353-376

Lampe JF, Burman O, Würbel $\mathrm{H}$ et al (2017) Context-dependent individual differences in playfulness in male rats. Dev Psychobiol 59:460-472

Lee PC, Moss CJ (2014) African elephant play, competence and social complexity. Anim Behav Cogn 1(2):144-156

Lutz MC, Judge PG (2017) Self-handicapping during play fighting in capuchin monkeys (Cebus apella). Behaviour 154:909-938

Lutz MC, Ratsimbazafy J, Judge PG (2019) Use of social network models to understand play partner choice strategies in three primate species. Primates 60(3):1-14

Maestripieri D (2004) Maternal behavior, infant handling, and socialization. In: Thierry B et al (eds) Macaque societies: a model for the study of social organization. Cambridge University Press, Cambridge, pp 231-234

Maestripieri D, Ross SR (2004) Sex differences in play among western lowland gorilla (Gorilla gorilla gorilla) infants: Implications for adult behavior and social structure. Am J Phys Anthropol 123:52-61

Mancini G, Palagi E (2009) Play and social dynamics in a captive herd of gelada baboons (Theropithecus gelada). Behav Process 82:286-292

Martin P, Caro TM (1985) On the functions of play and its role in behavioral development. In: Rosenblatt JS, Beer C, Busnel MC, Slater PJB (eds) Advances in the study of behavior, vol 15. Academic, New York, pp 59-103

Mather JA, Anderson RC (1999) Exploration, play and habituation in octopuses (Octopus dofleini). J Comp Psychol 113(3):333-338

Matsumura S (1999) The evolution of "egalitarian" and "despotic" social systems among macaques. Primates 40(1):23-31

Mayhew JA (2013) Attention cues in apes and their role in social play behavior of western lowland gorillas (Gorilla gorilla gorilla). PhD Thesis, The University of St Andrews, St Andrews, Scotland 
Nahallage CAD, Leca JB, Huffman MA (2016) Stone handling, an object play behaviour in macaques: welfare and neurological health implications of a bio-culturally driven tradition. Behaviour 153(6-7):845-869

Neumann C, Duboscq J, Dubuc C et al (2011) Assessing dominance hierarchies: validation and advantages of progressive evaluation with Elo-rating. Anim Behav 82:911-921

Ortega JC, Bekoff M (1986) Avian play: comparative evolutionary and developmental trends. Auk 104:338-341

Palagi E (2006) Social play in bonobos (Pan paniscus) and chimpanzees (Pan troglodytes): implications for natural social systems and interindividual relationships. Am J Phys Anthropol 129:418-426

Palagi E (2009) Adult play fighting and potential role of tail signals in ringtailed lemurs (Lemur catta). J Comp Psychol 123(1):1-9

Palagi E (2018) Not just for fun! Social play as a springboard for adult social competence in human and non-human primates. Behav Ecol Sociobiol 72:90

Palagi E, Antonacci D, Cordoni G (2007) Fine-tuning of social play in juvenile lowland gorillas (Gorilla gorilla gorilla). Dev Psychobiol 49(4):443-445

Panksepp J, Beatty WW (1980) Social deprivation and play in rats. Behav Neural Biol 30 (2): $197-206$

Pellis SM, Iwaniuk AN (2000) Comparative analyses of the role of postnatal development on the expression of play fighting. Ethology 106(12):1083-1104

Pellis SM, McKenna M (1992) What do rats find rewarding in play fighting? An analysis using drug-induced non-playful partners. Behav Brain Res 68(1):65-73

Pellis SM, Pellis VC (1996) On knowing it's only play: the role of play signals in play fighting. Aggress Violent Behav 1(3):249-268

Pellis SM, Pellis VC (2009) The playful brain. OneWorld Publications, Oxford

Pellis SM, Pellis VC, Reinhart CJ et al (2011) The use of the bared-teeth display during play fighting in Tonkean macaques (Macaca tonkeana): sometimes it is all about oneself. J Comp Psychol 125(4):393-403

Pellis SM, Pellis VC, Pelletier A et al (2019) Is play a behavior system, and, if so, what kind? Behav Process 160:1-9

Petit O, Bertrand F, Thierry B (2008) Social play in crested and Japanese macaques: testing the covariation hypothesis. Dev Psychobiol 50(4):399-407

Pruitt JN, Burghardt GM, Riechert SE (2012) Non-conceptive sexual behavior in spiders: a form of play associated with body condition, personality type, and male. Ethology 118:33-40

Reinhart CJ, Pellis V, Thierry B et al (2010) Targets and tactics of play fighting: competitive versus cooperative styles of play in Japanese and Tonkean macaques. Int J Comp Psychol 23 (2): 166-200

Scopa C, Palagi E (2016) Mimic me while playing! Social tolerance and rapid facial mimicry in macaques (Macaca tonkeana and Macaca fuscata). J Comp Psychol 130(2):153-161

Shimada M (2006) Social object play among young Japanese macaques (Macaca fuscata) in Arashiyama, Japan. Primates 47:342-349

Shimada M (2010) Social object play among juvenile Japanese macaques. In: Nakagawa $\mathrm{N}$ et al (eds) The Japanese macaques. Springer, New York, pp 375-385

Shimada M, Sueur C (2014) The importance of social play network for infant or juvenile wild chimpanzees at Mahale Mountains National Park, Tanzania. Am J Primatol 76(11):1025-1036

Shimada M, Sueur C (2017) Social play among juvenile wild Japanese macaques (Macaca fuscata) strengthens their social bonds. Am J Primatol 80(1):e22728

Siviy S, Deron L, Kasten C (2011) Serotonin, motivation, and playfulness in the juvenile rat. Dev Cogn Neurosci 1:606-616

Smith-Aguilar SE, Aureli F, Busia L et al (2018) Using multiplex networks to capture the multidimensional nature of social structure. Primates 60(3):1-19

Sueur C, Jacobs A, Amblard A et al (2011a) How can social network analysis improve the study of primate behavior? Am J Primatol 3:703-719 
Sueur C, Petit O, De Marco A et al (2011b) A comparative network analysis of social style in macaques. Anim Behav 82:845-852

Thierry B (1985) Patterns of agonistic interactions in three species of macaque (Macaca mulatta, M. fascicularis, M. tonkeana). Aggress Behav 11(3):223-233

Thierry B (1990) Feedback loop between kinship and dominance: the macaque model. J Theor Biol 145(4):511-521

Thierry B (2007) Unity in diversity: lessons from macaque societies. Evol Anthropol 16 (6):224-238

Thierry B (2011) The macaques: a double-layered social organization. In: Campbell CJ et al (eds) Primates in perspective, 2nd edn. Oxford University Press, New York, pp 229-241

Thierry B, Iwaniuk AN, Pellis SM (2000) The influence of phylogeny on the social behaviour of macaques (Primates: Cercopithecidae, genus Macaca). Ethology 106(8):713-728

Thompson KV (1996) Play-partner preferences and the function of social play in infant sable antelope, Hippotragus niger. Anim Behav 52:1143-1155

van Leeuwen EJC, Zimmerman E, Davila Ross M (2011) Responding to inequities: gorillas try to maintain their competitive advantage during play fights. Biol Lett 7:39-42

Ward C, Bauer E, Smuts B (2008) Partner preferences and asymmetries in social play among domestic dog, Canis lupus familiaris, littermates. Anim Behav 76(4):1187-1199

Whitehead H (2008) Analyzing animal societies: quantitative methods for vertebrate social analyses. University of Chicago Press, Chicago, IL

Wright KR, Mayhew JA, Sheeran LK et al (2018) Playing it cool: characterizing social play, bout termination, and candidate play signals of juvenile and infant Tibetan macaques (Macaca thibetana). Zool Res 39(4):1-13

Xia DP, Li JH, Garber P et al (2012) Grooming reciprocity in female Tibetan macaques Macaca thibetana. Am J Primatol 74(6):569-579

Xia DP, Li JH, Garber P et al (2013) Grooming reciprocity in male Tibetan macaques. Am J Primatol 75:1009-1020

Yamanashi Y, Nogami E, Teramoto M et al (2018) Adult-adult social play in captive chimpanzees: is it indicative of positive animal welfare? Appl Anim Behav Sci 199:75-83

Yanagi A, Berman CM (2014a) Functions of multiple play signals in free-ranging juvenile rhesus macaques (Macaca mulatta). Behaviour 151:1983-2014

Yanagi A, Berman CM (2014b) Body signals during social play in free-ranging rhesus macaques (Macaca mulatta): a systematic analysis. Am J Primatol 76:168-179

Zhao QK (1997) Intergroup interactions in Tibetan macaques at Mt. Emei, China. Am J Phys Anthropol 104(4):459-470

Open Access This chapter is licensed under the terms of the Creative Commons Attribution 4.0 International License (http://creativecommons.org/licenses/by/4.0/), which permits use, sharing, adaptation, distribution and reproduction in any medium or format, as long as you give appropriate credit to the original author(s) and the source, provide a link to the Creative Commons licence and indicate if changes were made.

The images or other third party material in this chapter are included in the chapter's Creative Commons licence, unless indicated otherwise in a credit line to the material. If material is not included in the chapter's Creative Commons licence and your intended use is not permitted by statutory regulation or exceeds the permitted use, you will need to obtain permission directly from the copyright holder.

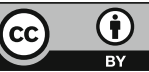

